\title{
DISTRIBUCIÓN DE AGUA DE RIEGO LIMITADA MEDIANTE ESTRATEGIAS DE RIEGO DEFICITARIO CONTROLADO OPTIMIZADO Y AÑO METEOROLÓGICO TÍPICO EN CEBOLLA
}

\author{
Leite, K.N. ${ }^{1}$, Pardo, J.J. ${ }^{2}$, Martínez-Romero, A. ${ }^{3}$, Tarjuelo, J.M. ${ }^{4}$, Domínguez, A. $^{5}$
}

${ }^{1}$ Coordenação de Aperfeiçoamento de pessoal do nivel superior (CAPES), Quadra 2, Bloco L, Lote 06, 70040-020, Brasilia, DF, Brazil; kellyleyte14@hotmail.es

${ }^{2}$ Ingeniero Agrónomo, Investigador; Centro Regional de Estudios del Agua (CREA), UCLM, Ctra. de Las Peñas km. 3.2, 02071 Albacete; josejesus.pardo@uclm.es

${ }^{3}$ Dr. Ingeniero Agrónomo, Profesor Asociado; ElIAB, Departamento de Mecánica Aplicada e Ingeniera de Proyectos, UCLM; angel.mromero@uclm.es

3 Dr. Ingeniero Agrónomo, Catedrático de Universidad; Departamento de Producción Vegetal y Tecnología Agraria, UCLM; jose.tarjuelo@uclm.es

${ }^{5}$ Dr. Ingeniero Agrónomo, Profesor Contratado Doctor; Departamento de Producción Vegetal y Tecnología Agraria, UCLM; alfonso.dominguez@uclm.es

\section{Resumen}

Bajo condiciones de limitación estricta del volumen de agua de riego, se debe evitar un agotamiento de éste en una etapa del desarrollo del cultivo temprana, ya que puede acarrear una disminución considerable en el rendimiento.

El objetivo de este trabajo ha sido desarrollar una metodología capaz de maximizar el rendimiento de un cultivo de cebolla cuando la cantidad de agua de riego disponible es inferior a las necesidades netas del cultivo para un año típico (In $=743.3 \mathrm{~mm}$ ). Para la validación de esta metodología se han considerado tres volúmenes de agua disponible $(0,9$ In, 0,8 In, y 0,7 In), simulando las estrategias de riego deficitario con el modelo MOPECO para la serie climática 2005-2012. La metodología propuesta combina dos metodologías existentes, el año meteorológico típico (TMY) y el riego deficitario optimizado por etapas (ORDI). Las simulaciones pusieron de manifiesto que la metodología logró una buena distribución dl agua disponible a lo largo de la campaña, a pesar de desconocer las condiciones climáticas en las que iba a desarrollar el cultivo. Los rendimientos obtenidos fueron alrededor de un $5.1 \%$ menores a los estimados al inicio de la campaña. Sólo 3 de los 24 escenarios analizados alcanzaron un déficit excesivo durante la última etapa, al agotar el agua antes de tiempo, por lo que la metodología puede utilizarse para distribuir volúmenes limitados de agua en un cultivo de cebolla del modo más eficiente.

\section{1- Introducción y Objetivos}

En zonas con escasez de agua, como es el caso de la unidad hidrogeológica "Mancha Oriental", las autoridades que gestionan y regulan los aprovechamientos hídricos limitan la cantidad de agua de riego que pueden usar los agricultores. Por tanto, se corre el riesgo de que en años secos se gaste prematuramente dicha cantidad de agua, provocando una caída significativa del rendimiento del cultivo y, en consecuencia, de la rentabilidad final. Si la disponibilidad de agua es además inferior a las necesidades netas del cultivo, el riesgo es todavía mayor.

Una adecuada distribución de esa cantidad de agua es esencial para minimizar o evitar el riesgo antes mencionado. Para ello se requiere una metodología que permita estimar las condiciones climáticas que van a afectar al cultivo durante la campaña de riegos y que oriente sobre la estrategia de riegos más conveniente. 
Si se dispone de un volumen limitado de agua de riego pueden plantear tres escenarios: 1) las necesidades de agua de un cultivo son similares a las de un año medio, y por tanto, se usará toda el agua disponible sin que haya estrés por déficit hídrico en el cultivo (año típico caracterizado como Intermedio); las necesidades de agua son mayores que la cantidad de agua disponible, pudiendo agotar el agua prematuramente, que conllevaría pérdidas considerables de rendimiento (condiciones climáticas caracterizadas como un año típico Seco); 3) las necesidades del cultivo son inferiores al volumen disponible y sobra agua (año típico caracterizado como húmedo).

El objetivo de este trabajo ha sido desarrollar una metodología capaz de maximizar el rendimiento de un cultivo de cebolla cuando la cantidad de agua de riego disponible es inferior a las necesidades netas del cultivo para un año típico (In $=743.3 \mathrm{~mm}$ ). Para la validación de esta metodología se han considerado tres volúmenes de agua disponible (0.9 In, $0.8 \mathrm{ln}$, y $0.7 \mathrm{ln}$ ), simulando las estrategias de riego deficitario con el modelo MOPECO (Ortega et al., 2004). La metodología propuesta combina dos metodologías existentes, el año meteorológico típico (TMY) (Domínguez et al., 2013) y el riego deficitario optimizado por etapas (ORDI) (Domínguez et al., 2012a).

\section{2- Materiales y métodos}

Utilizando los datos diarios de la estación climática "Los Llanos" (Albacete) para la serie 1951-2004, se han determinado tres tipos de TMY (seco, intermedio y húmedo) (Domínguez et al., 2013) en función del índice de déficit de precipitación (PD) mediante estas tres ecuaciones (Ec 1) (Leite et al., 2015):

$$
P D=P-\text { ETo } \quad Z=\frac{P D-P D \text { medio }}{D S} \quad R=\frac{\text { máximo } Z \text {-mínimo } Z}{n^{\circ} \text { de grupos }}
$$

Siendo DS la desviación estándar y quedando divididos los 3 grupos de la siguiente manera: Años secos: mínimo $Z \leq Z \leq$ mínimo $Z+R$.

Años intermedios: mínimo $Z+R \leq Z \leq$ máximoZ $-R$.

Años húmedos: máximoZ $-R \leq Z \leq$ máximoZ.

A partir de los datos climáticos del TMY intermedio se determinó la cantidad de agua de riego neta para cubrir las necesidades del cultivo (In $=743.3 \mathrm{~mm}$ ), y se obtuvieron los volúmenes netos disponibles para tres escenarios $(0.9 \mathrm{ln}, 0.8 \mathrm{In}$, y $0.7 \mathrm{In})$.

Para establecer la programación de riegos optimizada, se estimó la relación ETa/ETm para cada etapa de desarrollo fenológico del cultivo que maximizaba el rendimiento dada una relación $\mathrm{ETa/ETm}$ global (Domínguez et al., 2012a). Para ello es necesario conocer: la disponibilidad de agua de riego, el rendimiento potencial de la cebolla en la zona (Ym), la evapotranspiración potencial (ETm) acumulada teórica en cada una de las 4 etapas de diferente sensibilidad al déficit $(\mathrm{Ky})$ consideradas (establecimiento (i'), desarrollo vegetativo (i”), bulberización (ii), y maduración (iii)), los valores de Ky de cada etapa, y la precipitación efectiva de cada etapa de $\mathrm{Ky}(\mathrm{Pe})$ (Stewart et al., 1977).

$$
Y_{a}=Y_{m} \prod_{k=1}^{n=4}\left(1-k_{y_{k}}\left(1-\frac{E T_{a_{k}}}{E T_{m_{k}}}\right)\right)
$$

La ecuación y los coeficientes de estrés en cebolla fueron validados por Dominguez et al. (2012b) en Motilleja (Albacete) para relaciones ETa/ETm de entre 1 y 0.4 .

Para evitar resultados agronómicamente inaceptables y una posible sobreestimación del rendimiento, se introdujeron algunas restricciones en el optimizador, tales como limitar a 0.5 el mínimo valor que puede alcanzar la relación ETa/ETm objetivo en cada etapa (Doorenbos 
y Kassam, 1979), limitar a 0.8 el máximo estrés durante la etapa de establecimiento para garantizar una adecuada nascencia, debiendo determinar la máxima diferencia de ETa/ETm entre etapas de desarrollo consecutivas (Dominguez et al., 2012b).

La metodología seguida para determinar la cantidad de agua de riego a aplicar en cada una de las etapas de desarrollo de la cebolla puede resumirse en tres pasos:

1. 1a Optimización: En base a los datos climáticos de los meses previos a la siembra (desde el 1 de septiembre), se caracteriza el año en curso como seco, intermedio o húmedo. Los datos climáticos correspondientes al TMY de similar categoría al año actual son utilizados por la metodología ORDI para establecer las relaciones ETa/ETm objetivo para cada etapa Ky del ciclo en función de la disponibilidad de agua de riego, las necesidades de riego teóricas en cada etapa, y el rendimiento esperado para esas condiciones.

2. $2^{a}$ Optimización: Lograr la relación ETa/ETm objetivo determinada para la primera etapa en la primera optimización utilizando los datos climáticos reales del año en curso, probablemente implicará haber utilizado una cantidad de agua de riego diferente a la prevista en la $1^{\text {a }}$ optimización. También serán diferentes la ETm y la precipitación efectiva $(\mathrm{Pe})$ acumuladas. En consecuencia, es necesario realizar una nueva optimización que determine las relaciones ETa/ETm objetivo para el resto de etapas teniendo en cuenta la nueva disponibilidad de agua de riego y la relación $\mathrm{ETa} / \mathrm{ETm}$ realmente obtenida en la primera etapa. Además, se analiza si el año continúa siendo seco, húmedo o intermedio. En esta $2^{a}$ Optimización se reserva cierta cantidad de agua para la última etapa (relación ETa/ETm de la cuarta etapa en la primera optimización multiplicada por la ETm esperada de esa etapa).

3. $3^{\mathrm{a}}$ y $4^{\mathrm{a}}$ Optimización: Se repite el paso 2 para las etapas de Ky 3 y 4 .

\section{3- Resultados y Discusión.}

Con los datos climáticos de los años 2005 a 2012 se calculó la distribución óptima del agua de riego disponible para cada etapa de desarrollo fenológico para 3 dotaciones de agua ( 0.9 In, $0.8 \mathrm{ln}$, y $0.7 \mathrm{ln}$, siendo In las necesidades netas de riego para el "Año intermedio"). Siguiendo la metodología propuesta, al inicio del proceso de optimización se determinó el tipo de TMY, y así sucesivamente para cada etapa de desarrollo fenológico (Tabla 1).

Tabla 1. Evolución del tipo de año durante la campaña de riegos y relación $P D_{T M Y} / P D_{\text {actuall }}$.

\begin{tabular}{|c|c|c|c|c|c|c|c|c|}
\hline Optimization & 2005 & 2006 & 2007 & 2008 & 2009 & 2010 & 2011 & 2012 \\
\hline \multirow[t]{2}{*}{ 1st } & Dry & Inter. & Inter. & Dry & Wet & Inter. & Wet. & Dry \\
\hline & 0.82 & 0.87 & 0.89 & 0.87 & 0.98 & 1.09 & 0.90 & 1.16 \\
\hline \multirow[t]{2}{*}{ 2nd } & Dry & Inter. & Inter. & Dry & Wet & Inter. & Wet & Dry \\
\hline & 0.83 & 1.15 & 1.17 & 1.03 & 0.86 & 1.07 & 0.92 & 0.95 \\
\hline \multirow[t]{2}{*}{$3 \mathrm{rd}$} & Dry & Inter. & Inter. & Inter. & Inter & Inter. & Wet & Dry \\
\hline & 0.79 & 1.11 & 1.06 & 1.20 & 1.03 & 1.09 & 0.93 & 0.93 \\
\hline \multirow[t]{2}{*}{ 4th } & Dry & Inter. & Inter. & Dry & Dry & Inter. & Inter & Dry \\
\hline & 0.83 & 1.05 & 0.98 & 1.04 & 1.00 & 0.99 & 1.03 & 0.92 \\
\hline \multirow[t]{2}{*}{ End } & Dry & Inter. & Inter. & Dry & Dry & Inter. & Inter & Dry \\
\hline & 0.85 & 1.03 & 0.96 & 1.01 & 1.05 & 1.04 & 1.12 & 0.95 \\
\hline
\end{tabular}

Donde: Dry, seco; Inter., intermedio; Wet, húmedo.

La mayoría de los años mantuvieron la misma calificación durante toda la campaña. Sin embargo, los años 2009 y 2011 evolucionaron a situaciones más desfavorables, pasando de un año considerado húmedo a seco e intermedio, respectivamente. Lo ocurrido en 2009 es el peor escenario posible. El modelo asume que dispondrá de una mayor disponibilidad de agua de lluvia, pudiendo aportar más agua de riego en las etapas iniciales, precisamente en las que el ahorro de agua de riego tiene un menor efecto en el rendimiento final. Esto se tradujo en una falta de agua durante la etapa de maduración, en parte amortiguada por la reserva de agua de la segunda optimización. La relación $\mathrm{PD}_{\mathrm{TMY}} / \mathrm{PD}_{\text {actual }}$ se utilizó para determinar como de seco, intermedio o húmedo era el año actual. Como ejemplo, 2005 fue más seco que el TMY-seco y 2011 fue el más húmedo, pero ligeramente más seco que un 
TMY-húmedo. Gracias a las sucesivas optimizaciones al finalizar cada etapa de desarrollo, así como reservar una cierta cantidad de agua para la última etapa, los efectos de la variabilidad climática son minimizados.

En la mayoría de los casos el modelo consiguió alcanzar la relación ETa/ETm objetivo en cada una de las etapas (Fig. 1A). Sin embargo, en los años más secos y con menor disponibilidad de agua puede apreciarse como la última etapa es la que se queda por debajo del objetivo (años 2007 y 2009). En algunas etapas, donde la lluvia fue superior a la esperada, incluso se obtuvieron relaciones ETa/ETm superiores a la objetivo (2008 y 2010).

Para lograr las relaciones ETa/ETm objetivo el modelo tuvo que aplicar dosis de riego que en ocasiones fueron muy diferentes a las esperadas en cada etapa (Fig. 1B). Esto fue debido principalmente a la irregular distribución de la lluvia (que no coincide con la del año típico utilizado) y en menor medida a las variaciones diarias de ETo.
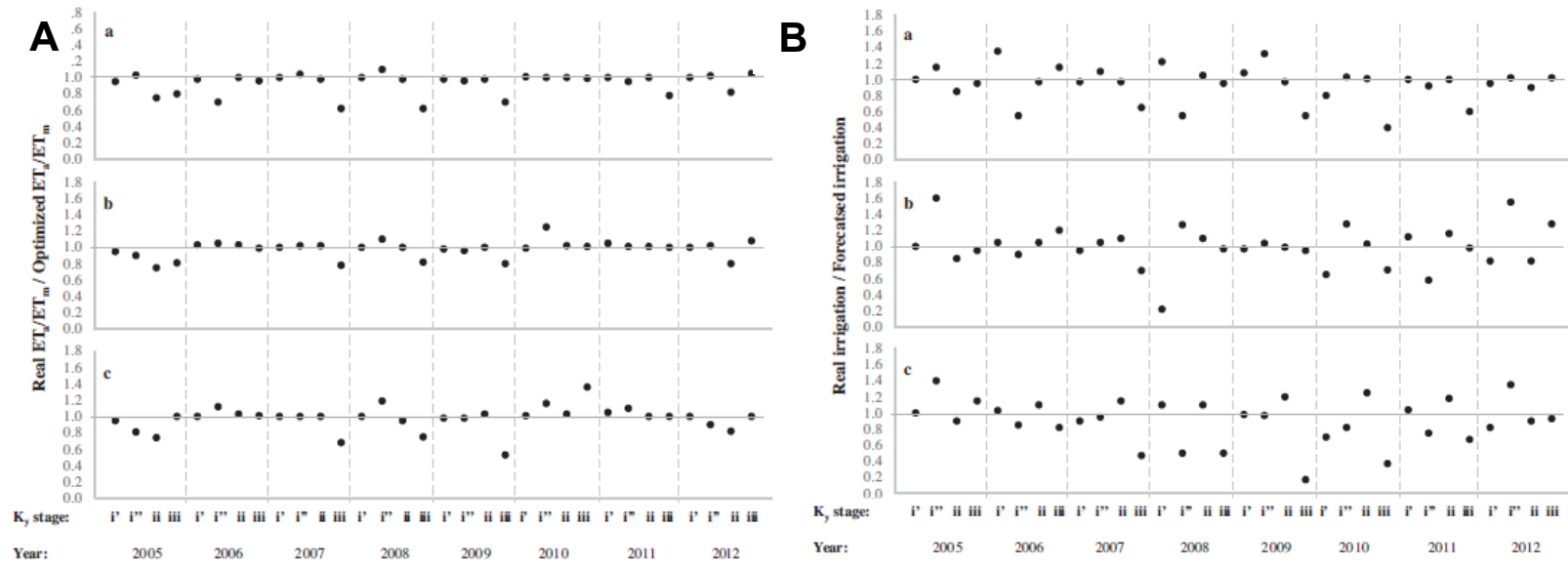

Figura 1.A) Relación entre la relación ETa/ETm real y la objetivo para los tres niveles de agua total disponible $(0.9 \mathrm{In}, 0.8 \mathrm{In}$ y $0.7 \mathrm{ln})$; 1B) Relación entre el riego real y el previsto para tres niveles de agua total disponible (a: $0.9 \mathrm{ln}, \mathrm{b}: 0.8 \mathrm{ln}, \mathrm{c}: 0.7 \mathrm{ln}$, siendo $\mathrm{ln}: 743.3 \mathrm{~mm}$ ).

Los rendimientos esperados al inicio de la campaña (primera optimización) se compararon con el rendimiento final obtenido (Fig. 2). La mayoría de los rendimientos estimados al finalizar el año han variado un $\pm 5 \%$ con respecto a los previstos al inicio de la campaña, aunque en los escenarios más desfavorables $(2005,2009$ y 2012) han sido hasta un $10-15 \%$ inferiores (Fig. 2). Sólo 3 de los 24 escenarios analizados alcanzaron un déficit excesivo durante la última etapa, al agotar el agua antes de tiempo, por lo que la metodología puede utilizarse para distribuir volúmenes limitados de agua en un cultivo de cebolla del modo más eficiente. Pese a todo, la metodología propuesta puede ser adecuada para asesorar a los regantes sobre la manera de mejorar la distribución del agua de riego durante el periodo de crecimiento bajo condiciones de déficit moderado ( 0.9 y $0.8 \mathrm{In})$.

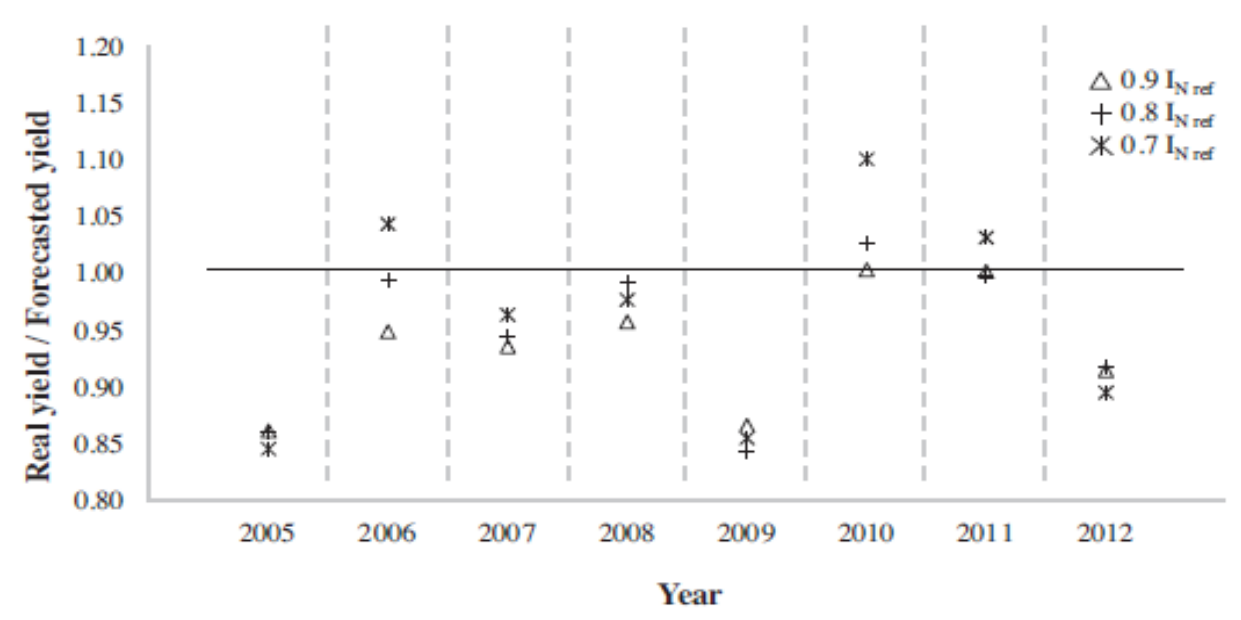


Figura 2. Relación entre el rendimiento real y el previsto para los tres niveles de agua total disponible (0.9 In, $0.8 \ln$ y $0.7 \ln )$.

Se comparó en MOPECO la metodología propuesta con un riego deficitario en el que el nivel de déficit se mantuvo constante en todas las etapas (CDI). La cantidad de agua disponible fue 0.8 In para el caso de ORDI, y la relación objetivo $\mathrm{ETa} / \mathrm{ETm}=0.8$ para el $\mathrm{CDI}$. El año elegido fue 2010 (intermedio) (Fig.3).

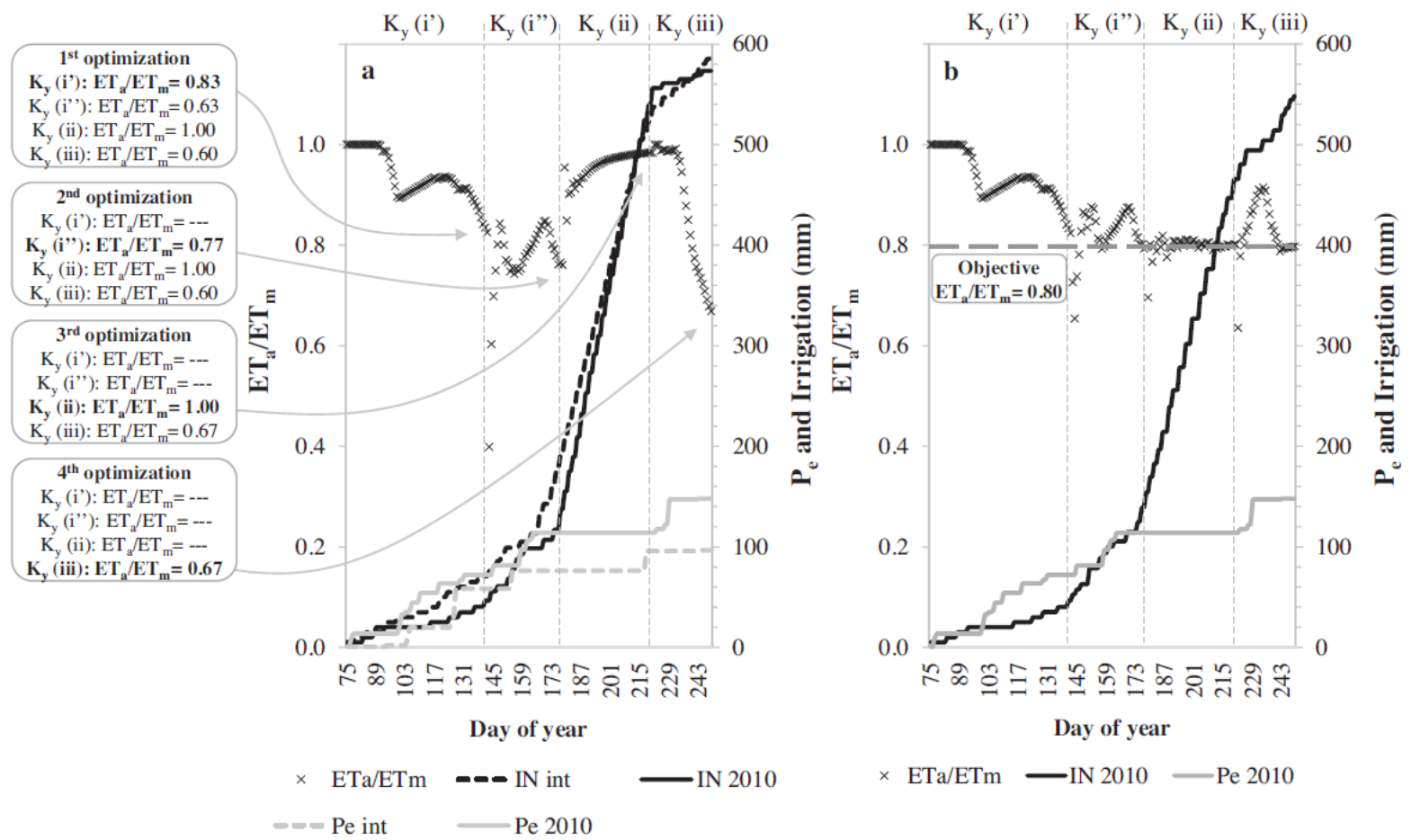

Figura 3. Comparación de la distribución del agua de riego entre el optimizado (a) y el CDI

(b) para el año 2010 y 0.8 In de volumen de agua disponible; Ky: etapa de desarrollo;

ETa/ETm: relación entre la evapotranspiración real y máximo acumulado en cada etapa; Pe: lluvia efectiva acumulada en condiciones de TMY-intermedio (Peint), y en condiciones reales del año 2010 (Pe2010); IN: progresión neta de riego acumulado [prevista para las condiciones meteorológicas de TMY-intermedio (IN int), y simulado por MOPECO para el año 2010 (IN2010)].

Como se aprecia en la figura 3, debido a las lluvias de la última etapa, el agua empleada en el CDI fue un $4.5 \%$ menor que en el ORDI (548.5 frente a $573.4 \mathrm{~mm}$ ). Sin embargo, el rendimiento simulado obtenido por el CDI fue un $13.2 \%$ menor al ORDI (73542 kg ha ${ }^{-1}$ frente a $83510 \mathrm{~kg} \mathrm{ha}^{-1}$ ). Estas diferencias implican una diferencia de margen bruto de $627.4 € \mathrm{ha}^{-1}$ a favor del ORDI.

\section{3- Conclusiones y recomendaciones}

La metodología propuesta en este estudio, que combina las técnicas ORDI y TMY, permite a los agricultores reducir al mínimo las pérdidas de rendimiento en el cultivo de cebolla (alrededor de $5.1 \%$ respecto al rendimiento teórico esperado al inicio de la campaña para el volumen de agua de riego disponible) mediante la optimización de la distribución del agua de riego durante todo el ciclo de cultivo. Además, se consigue un uso más eficiente del agua y un margen bruto mayor (13.2\%) comparado con un riego deficitario constante por etapas. 
Las sucesivas optimizaciones al finalizar cada etapa de desarrollo, así como reservar una cierta cantidad de agua para la última etapa, permiten minimizar los efectos de la variabilidad climática.

\section{4- Bibliografía.}

Domínguez, A., de Juan, J.A., Tarjuelo, J.M., Martínez, R.S., Martínez-Romero, A., 2012a. Determination of optimal regulated deficit irrigation strategies for maize in a semi-arid environment. Agric. Water Manage. 110, 67-77.

Domínguez, A., Jiménez, M., Tarjuelo, J.M., de Juan, J.A., Martínez-Romero, A., Leite,K.N., 2012b. Simulation of onion crop behaviour under optimized regulated deficit irrigation using Mopeco model in a semi-arid environment. Agric. WaterManage. 113, 64-75.

Domínguez, A., Martínez-Romero, A., Leite, K.N., Tarjuelo, J.M., de Juan, J.A., LópezUrrea, R., 2013. Combination of typical meteorological year with regulateddeficit irrigation to improve the profitability of garlic in central Spain. Agric.Water Manage. 130, 154-167.

Doorenbos, J., Kassam, A.H., 1979. Yield response to water. In: Irrigation and DrainagePaper No. 33. FAO, Italy.

Leite, K.N., Martínez-Romero, A., Tarjuelo J.M., Domínguez, A., 2015. Distribution of limited irrigation water base don optimized regulated déficit irrigation and typical metheorological year concepts. Agric. Water Manage. 148, 164-176.

Microsoft, 2010. Microsoft Excel 2010. Product Guide. Microsoft Corp., USA.

Ortega, J.F., de Juan, J.A., Martín-Benito, J.M., López-Mata, E., 2004. MOPECO: an economic optimization model for irrigation water management. Irrig. Sci. 23 (2), 61-75.

Stewart, J.I., Hagan, R.M., Pruitt, W.O., Kanks, R.J., Riley, J.P., Danilson, R.E., Franklin,W.T., Jackson, E.B., 1977. Optimizing crop production through control of waterand salinity levels. Utah Water Res. Lab. PWRG 151-1, Utah, USA. 